\title{
Changes in cartilage proteoglycan aggrecan after intra-articular injection of interleukin-1 in rabbits: studies of synovial fluid and articular cartilage
}

\author{
Claes Lundberg, Inger Åsberg, Mirela Ionescu, Agnes Reiner, Göran Smedegård, \\ A Robin Poole
}

Department of Pharmacology, Pharmacia Pharmaceutials Uppsala, S-751 82 Uppsala, Sweden C Lundberg I Åsberg

G Smedegård

Joint Diseases

Laboratory, Shriners

Hospital for Crippled Children, Division of Surgical Research, Department of Surgery, McGill University, 1529 Cedar Avenue, Montreal, Quebec, Canada, H3G 1 A6

$M$ Ionescu

A Reiner

A R Poole

Correspondence to:

Dr C Lundberg,

Department of

Pharmacology,

Pharmacia Pharmaceuticals Pharmacia

Uppsala,
S-751 82 Uppsala, Sweden

Accepted for publication

11 April 1996

\begin{abstract}
Objectives-To determine how acute but transient inflammation affects the cartilage proteoglycan aggrecan and the value of analyses of synovial fluid to study this. Methods-For 96 hours after a single intra-articular injection of rabbit knees

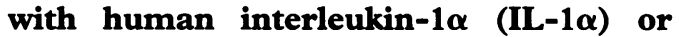
vehicle, articular cartilage and synovial fluid were examined using a putative indicator of aggrecan synthesis (aggrecan chondroitin sulphate epitope 846), immunoreactive keratan sulphate, and total glycosaminoglycan (GAG) content. Aggrecan extractability (with $0.5 \mathrm{M} \mathrm{NaCl}$ ) followed by $4 \mathrm{M}$ guanidine hydrochloride extraction permitted analyses of cartilage damage, total content and aggrecan heterogeneity. Aggrecan epitopes as well as GAG were assayed in synovial fluid. Changes were related to total joint leucocyte content in synovial fluid.
\end{abstract}

Results-At $10 \mathrm{ng}$, IL-1 $\alpha$ produced a transient increase in synovial fluid leucocytes at six hours and 24 hours. This accompanied a reduction in content and increased extractability of GAG, which was greatest in the tibial medial compartment of the knee. Further studies of this compartment showed no change in keratan sulphate epitope content, but a transient increase in extractability in 0.5 $\mathrm{M} \mathrm{NaCl}$. Epitope 846 content and extractability were unchanged. Total contents and extractability for GAG were inversely correlated in both controls and joints injected with IL-1 $\alpha$. These changes were accompanied by transient increases in GAG, keratan sulphate epitope, and 846 content in synovial fluid.

Conclusion-According to the aggrecan component measured, damage to the matrix of articular cartilage was sometimes reflected by a transient increased extractability and a net loss of aggrecan. There was always an increased release of GAG, and keratan sulphate, and 846 epitopes into synovial fluid. These studies show that changes in aggrecan epitopes and GAG in synovial fluid reflect changes in cartilage metabolism induced by acute transient inflammation.

(Ann Rheum Dis 1996; 55: 525-534)
Articular cartilage is characterised by an extensive extracellular matrix, which contains a fibrillar network of collagen fibrils composed primarily of type II collagen. It also contains a large aggregating proteoglycan called aggrecan. Whereas collagen endows cartilage with its tensile strength, the unique compressive stiffness of cartilage is determined by these large proteoglycans. ${ }^{12}$

The core protein of aggrecan was cloned and sequenced by Doege et al. ${ }^{34}$ It consists of three globular domains, one of which, the G1 domain at the amino terminus, binds to hyaluronan and thereby retains these molecules within the extracellular matrix. Close to the G1 domain is a second globular domain G2. Situated in a region of the core protein close to $\mathrm{G} 2$ is a keratan sulphate rich region (absent in the rat and mouse) in which up to 30 of these glycosaminoglycan chains are located. Situated between this region and the carboxy terminal G3 globular domain is a chondroitin sulphate rich region containing up to 100 chains. ${ }^{12}$

In human cartilage, some chondroitin sulphate chains on the largest aggrecan molecules bear an epitope recognised by monoclonal antibody $846 .{ }^{56}$ These chains are thought to reside close to the G3 globular domain. ${ }^{6}$ Using monoclonal antibodies to $\mathrm{KS}$ and to the chondroitin sulphate epitope 846 , degradation products of these molecules, rich in keratan sulphate, relatively deficient in chondroitin sulphate, and lacking the 846 epitope, have been identified in normal articular cartilages during development and in aging. ${ }^{6-9}$ Based on detailed analyses of these molecules in human articular cartilages, we have proposed that the largest molecules bearing the 846 epitope may be recently synthesised. ${ }^{6}$ Molecules rich in keratan sulphate but lacking the 846 epitope, which accumulate in articular cartilage with development and age, ${ }^{9}$ may represent degradation products of molecules from which the 846 containing domain has been removed by proteolysis in the extracellular matrix. ${ }^{6}$

Studies of the degradation of these proteoglycans have shown that primary sites of cleavage reside within the core proteins of these molecules, leading to the release of degradation products. ${ }^{10-12}$ Although cleavage sites have been identified between the G1 and G2 globular domains. ${ }^{10-12}$ the accumulation of larger aggregating keratan sulphate rich degradation 
products in the matrix ${ }^{689}$ reflects the existence of other proteolytic sites.

Pettipher et $a l^{1314}$ have shown that intraarticular injection of interleukin-1 (IL-1) into rabbit knees causes cartilage degradation which is characterised by the release of proteoglycan containing chondroitin sulphate and immunoreactive karatan sulphate into synovial fluid. This is accompanied by a loss of proteoglycan from articular cartilage. Similar studies of the effects of IL-1 on cartilage proteoglycans have also been made in mice ${ }^{15}$ and rats, ${ }^{16}$ in which there is evidence that IL-1 also inhibits proteoglycan synthesis. Moreover, the inhibition of proteoglycan synthesis in antigen induced arthritis is also due in part to IL-1. ${ }^{17}$

In rheumatoid arthritis and osteoarthritis in humans, fragments of aggrecan molecules bearing a keratan sulphate epitope $\mathrm{e}^{18} 19$ and the 846 epitope $^{19}$ are detectable in synovial fluids of patients with chronic disease. In rheumatoid arthritis, concentrations of immunoreactive keratan sulphate are often increased with increasing polymorphonuclear leucocyte count, whereas the ratio of 846 to keratan sulphate epitopes is reduced under these conditions, suggesting impaired synthesis with enhanced degradation. ${ }^{19}$

In this study, we investigated, in more detail, the effect of IL- $1 \alpha$ with new immunochemical analyses of aggrecan changes which are now being used to study patients with arthritis. ${ }^{19} \mathrm{We}$ analysed different articular cartilages and showed that the most pronounced changes are in the medial tibial plateau. This, as well as synovial fluids, were selected for further study for proteoglycan aggrecan content measured both biochemically and using immunoassays for the 846 and keratan sulphate epitopes.

\section{Material and methods}

ANIMALS AND ANAESTHESIA

Male New Zealand white rabbits (body weight $2 \cdot 2-3 \cdot 1 \mathrm{~kg} ; 4-5$ months old) were purchased from Estuna, Sweden. The animals were anaesthetised intravenously with aplhaxoline/ alphadolone acetate $(9 \mathrm{mg} / \mathrm{kg}$, Saffan Glaxovet, Harefield, UK).

\section{ACUTE ARTHRITIS INDUCED BY IL- $1 \alpha$}

Human recombinant IL-1 $\alpha$ (specific activity 108 units/mg; Genzyme, Boston MA, USA) in $0.5 \mathrm{ml}$ phosphate buffered saline (PBS) containing $0 \cdot 1 \%$ bovine serum albumin (BSA), was injected randomly into the left or right joint of the rabbit knee, through the suprapatellar ligament. The contralateral knee joint was injected with an equal volume of the vehicle. The BSA solution was assayed for the presence of endotoxin using the Coatest endotoxin kit (Chromogenix, Mölndal, Germany). It was found to contain less than $0.27 \mathrm{ng} / \mathrm{ml}$. In the first experiment, 2,10 , and $50 \mathrm{ng} \mathrm{IL-1} \alpha /$ joint were injected intra-articularly ( $n=6$ per dose). The animals were killed 24 hours later. In the second experiment $10 \mathrm{ng}$ IL-1 $\alpha$ were injected per joint ( $n=4$ per time point). Rabbits were killed 6,24 , and 96 hours later.
COLLECTION OF SYNOVIAL FLUID AND ARTICULAR CARTILAGE

At the indicated time points, and after killing the rabbits, $1 \mathrm{ml}$ saline $(0.9 \%$ sodium chloride in water containing $0.38 \%$ sodium citrate) was injected intra-articularly into both the IL- $1 \alpha$ and control injected joint. The knee was flexed and extended 30 times, the joint was opened, and the joint lavage fluid was collected with a $1 \mathrm{ml}$ syringe with a cut off needle. Lavage fluid volume was estimated by weighing tubes and syringes. Total and differential leucocyte counts in the lavage fluid were determined. Joint lavage fluid was centrifuged at $5000 \mathrm{~g}$ for five minutes. Supernatants were aliquoted in $100 \mu \mathrm{l}$ volumes and frozen at $-70^{\circ} \mathrm{C}$ for later analysis. Total content of proteoglycan in joint fluids was expressed/joint. Dissection of the knee joints was performed using diathermia (Elektrotom, Mikron Med AB, Stockholm, Sweden) to avoid blood contamination. Articular cartilage was removed from the lateral and medial ends of the femur and tibia, weighed, and frozen.

\section{EXTRACTION OF CARTILAGE}

The cartilage samples were weighed (about 20 $\mathrm{mg}$ ), chopped with a scalpel, and successively extracted in $0.5 \mathrm{M} \mathrm{NaCl}, 4 \mathrm{M}$ guanidine hydrochloride ( $\mathrm{GuHCl})$, and papain as follows. The first extraction was in $2 \mathrm{ml} 0.5 \mathrm{M} \mathrm{NaCl}$ at $4^{\circ} \mathrm{C}$ overnight. After centrifugation at $1300 \mathrm{~g}$ for five minutes the supernatant was collected. For the second extraction $2 \mathrm{ml} 4 \mathrm{M} \mathrm{GuHCl}$ in $0.5 \mathrm{M}$ acetate buffer, $\mathrm{pH} 5.8$ was added to the pellets and extraction was performed as described above. After centrifugation under the same conditions the supernatants were collected. For the third extraction the pellets were washed once in $0.5 \mathrm{ml} 0.9 \% \mathrm{NaCl} ; 0.5 \mathrm{ml} 0.1 \mathrm{M}$ phosphate buffer, $\mathrm{pH} 6 \cdot 3$, was added to the pellet followed by $0.2 \mathrm{ml}$ papain solution ( 1.3 units $/ \mathrm{ml}$; Sigma No P3125, St Louis, MO, USA). The papain was dissolved in $0 \cdot 1 \mathrm{M}$ phosphate buffer $(\mathrm{pH}$ 6.3) with $0.5 \mathrm{M} \mathrm{NaCl}, 5 \mathrm{mM}$ EDTA, and $5 \mathrm{mM}$ cysteine hydrochloride. The samples were incubated at $65^{\circ} \mathrm{C}$ overnight and thereafter treated with $0.1 \mathrm{ml} 20 \mathrm{mM}$ iodoacetamide to inactivate the papain. The supernatants from the $\mathrm{GuHCl}$ extraction were desalted on Sephadex G25 (Pharmacia, Uppsala, Sweden).

\section{CHROMATOGRAPHY OF PROTEOGLYCANS ON} SEPHAROSE CL-2B

For analyses of proteoglycan by chromatography on Sepharose CL-2B (Pharmacia, Uppsala, Sweden), cartilages were directly extracted with $4 \mathrm{M} \mathrm{GuHCl}$ and proteinase inhibitors $^{6}$ as above. The extracts of normal articular cartilage were chromatographed in the presence of $4 \mathrm{M} \mathrm{GuHCl}$ as described previously ${ }^{6}$ to identify the different populations bearing the 846 and keratan sulphate epitopes.

\section{SYNOVIAL FLUIDS}

Papain digestion of joint lavage fluid was performed in the dose-response study before 
analysis of GAG. Lavage fluid $(50 \mu \mathrm{l})$ was mixed with $50 \mu 10 \cdot 1 \mathrm{M}$ phosphate buffer, $\mathrm{pH}$ $6.3,100 \mu l$ papain solution (see above) was added, and the mixture incubated at $65^{\circ} \mathrm{C}$ overnight. At the end of the incubation $50 \mu \mathrm{l}$ $20 \mathrm{mM}$ iodoacetate was added.

ANALYSIS OF SULPHATED GAGS, KERATAN SULPHATE, AND CHONDROITIN SULPHATE EPITOPE 846

The content of sulphated GAGs was determined using the 1,9-dimethylmethylene blue assay. ${ }^{20}$ Immunochemically reactive keratan sulphate was determined with a competitive inhibition solution phase radioimmunoassay, with intact purified rabbit costal cartilage proteoglycan as the standard. ${ }^{21}$ The same molecule was radiolabelled with ${ }^{125} \mathrm{I}$ and used with the univalent Fab fragment of the mouse monoclonal antibody 5D4. ${ }^{19}$ An immunoassay for the chondroitin sulphate epitope 846 of aggrecan was described recently: ${ }^{19}$ Here, purified human fetal proteoglycan is the standard. As the immunoassays employ different aggrecan standards, and these standards do not include rabbit articular aggrecan, it is not possible to determine the absolute contents of rabbit articular cartilage aggrecan molecules with these immunoassays.

\section{STATISTICAL METHODS}

Data are presented as mean (SD). The following statistical analysis was performed: an analysis of variance (ANOVA) model was fitted with the factors treatment and dose, or with the factors treatment and time with animals nested in dose and time. The factor treatment had two levels (IL- $1 \alpha$ and control), dose and time had three levels $(2,10$, and $50 \mathrm{ng}$ and 6,24 , and 96 hours after injection). If significant interaction effects were present, a paired $t$ test was used. Analysis of covariance (ANCOVA) between joints was performed together with correlations by regression analysis. For the variable GAG extractability the log linear doseresponse relation: $y=a+b x$, where $x=\ln$ (dose), was estimated for each compartment (medial and lateral tibia and femur). An ANCOVA was performed to test for heterogeneity of slopes.

\section{Results}

IDENTIFICATION OF PROTEOGLYCAN AGGRECAN SUBPOPULATIONS PRESENT IN RABBIT

ARTICULAR CARTILAGE

Rabbit articular cartilages from two healthy uninjected rabbits were isolated from medial and lateral sites of the tibia and femur, chopped, and directly extracted with $4 \mathrm{M}$ $\mathrm{GuHCl}$ (without prior extraction with $0.5 \mathrm{M}$ $\mathrm{NaCl}$ ). The extracts were then chromatographed on Sepharose CL-2B in $4 \mathrm{M} \mathrm{GuHCl}$ and analysed as described previously ${ }^{6}$ for 846 epitope, keratan sulphate epitope, and GAG content. Figure 1 shows the results. The 846 epitope was present on a population of proteoglycan molecules (the standard in the 846 assay

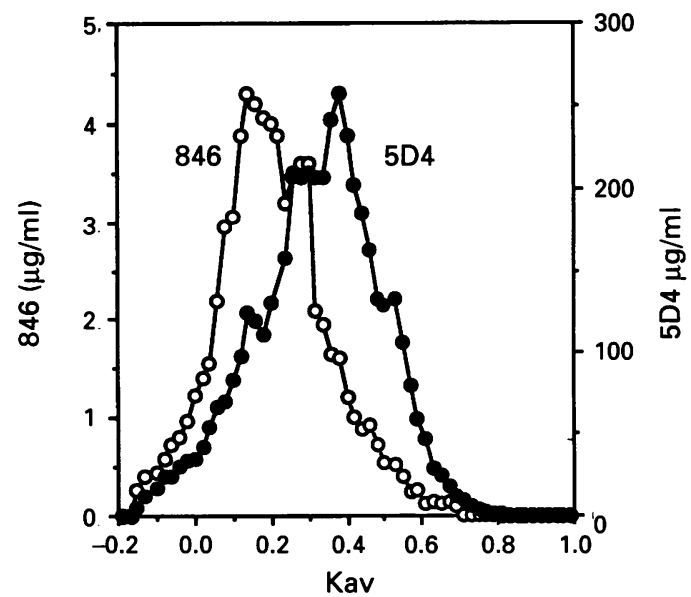

Figure 1 Aggrecan populations present in nomal rabbit articular cartilage. Chromatography of total $4 \mathrm{M} \mathrm{GuHCl}$ extract of normal cartilage on Sepharose $C L-2 B$ in the presence of $4 \mathrm{M} \mathrm{GuHCl}$. Column fractions were assayed for 846 and keratan sulphate (5D4) epitopes.

was human fetal proteoglycan) which also contained immunoreactive keratan sulphate measured with the 5D4 antibody (standard for 5D4 assay, adult rabbit proteoglycan) (approximate ratio 1:50). The keratan sulphate epitope eluted in a population with a larger Kav. The 846 epitope bearing molecules have a smaller Kav. They also represent similar diversity in size to that of the keratan sulphate epitope population (fig 1). Almost identical results were obtained with articular cartilages removed from joints treated with IL-1 $\alpha(10 \mathrm{ng} /$ joint for 24 hours; data not shown).

\section{TOTAL LEUCOCYTE CONTENT IN SYNOVIAL} FLUID

Effects of injection of IL-1 $\alpha$ at 2, 10, and 50 $\mathrm{ng} /$ joint were first examined after 24 hours. Significant increases in leucocyte content were dose dependent. They occurred at 10 and 50 ng compared with contralateral joints injected with vehicle alone (fig $2 \mathrm{~A}$ ). Further analysis of the $10 \mathrm{ng} /$ joint dose showed that the increase in leucocyte content was higher at six hours $\left(4.9 \times 10^{7}\right.$ cells), than at 24 hours, reducing to $0.2 \times 10^{6}$ cells at 96 hours (table; fig 2B). Polymorphonuclear leucocytes were the predominant cell at all time points and doses (data not shown). The contralateral joint injected with BSA alone had leucocyte numbers below $0.5 \times 10^{6}$ except at six hours when numbers had increased to $5.2 \times 10^{6}$ cells.

DOSE DEPENDENT CHANGES AT 24 HOURS AFTER INJECTION OF IL-1 $\alpha$ AT 2, 10, AND 50 ng PER JOINT

Aggrecan content and extractabilities of different articular cartilages

Cartilage analyses were made of lateral and medial compartments of the tibia and of femoral lateral and medial condyles at 24 hours, when cartilage changes were maximally defined as shown by other analyses (see below). Total GAG content was significantly reduced in all sites after injection of IL-1 $\alpha$ at 10 and 50 ng (fig 3). Only the medial tibial compartment 


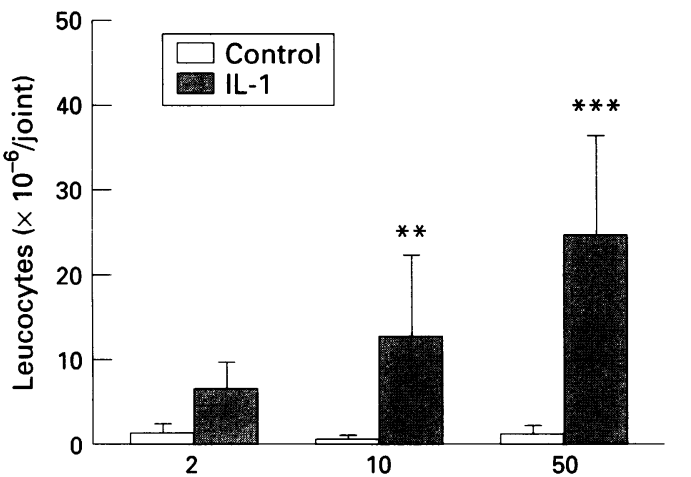

IL-1 (ng/joint)

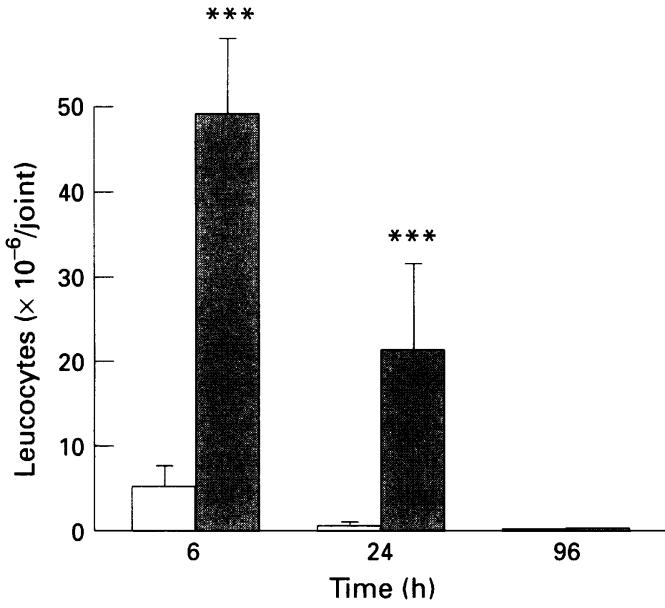

Figure 2 Accumulation of leucocytes (mean (SD)) in the synovial lavage fluid of the knee joint. Top, 24 hours after intra-articular injection of $B S A$ (control) or 2,10 , and 50 ng. IL-1 with BSA $(n=6)$; bottom, 6, 24, and 96 hours after intra-articular injection of $B S A$ (control, open box) or $10 \mathrm{ng} I L-1$ (closed box) $(n=4) .{ }^{*} p<0.01$; $\star * * p<0.001$.

showed a significant although small reduction in content at $2 \mathrm{ng}$ (fig 3A). Further analyses of extractability of total GAG showed that this was also significantly increased in all sites at 10 and $50 \mathrm{ng} \mathrm{IL-1} \alpha$. This increase was greatest in the medial tibial compartment (fig 4A-D). On statistical analysis, the medial tibial cartilage had the steepest estimated slope $(b=3 \cdot 3)$. It was thus the most sensitive compartment regarding variable GAG extractability. Moreover, only this compartment showed this effect of IL- $1 \alpha$ at $2 \mathrm{ng}$ (fig $4 \mathrm{~A}$ ). In view of these differences, the medial tibial compartment was selected for further study.

Summary of relative differences in joints injected with $I L-1 \alpha$ compared with contralateral joints (figs 6-8)

\begin{tabular}{llll}
\hline Marker/ocation & \multicolumn{2}{l}{ Time $(h)$} & \\
\cline { 2 - 4 } & 6 & 24 & 96 \\
\hline Leucocytes in synovial fluid & +++ & ++ & 0 \\
GAG: & & & \\
$\quad$ Total cartilage & 0 & 0 & - \\
$\quad$ Cartilage extractability & ++ & ++ & + \\
$\quad$ Total synovial fluid & ++ & +++ & 0 \\
Keratan sulphate: & & & \\
$\quad$ Total cartilage & 0 & 0 & 0 \\
$\quad$ Cartilage extractability & 0 & + & 0 \\
$\quad$ Total synovial fluid & ++ & +++ & 0 \\
846: & & & \\
$\quad$ Total cartilage & 0 & 0 & 0 \\
$\quad$ Cartilage extractability & 0 & 0 & 0 \\
$\quad$ Total synovial fluid & ++ & ++ & 0 \\
\hline
\end{tabular}

$0=$ no change $;+1++/+++=$ amount of increase $;-=$ decrease.
Analysis of immunoreactive keratan sulphate disclosed that, by contrast with total GAG content, there was no significant decrease in content of molecules bearing the keratan sulphate epitope after injection of IL- $1 \alpha$ at 2 , 10 , and $50 \mathrm{ng}$ (fig 5A). There was an increase in extractability of immunoreactive karatan sulphate, but only at 10 and $50 \mathrm{ng}$ (fig $5 \mathrm{~B}$ ), and not at $2 \mathrm{ng}$ as in the case of total GAG. Thus total GAG is not representative of those aggrecan molecules containing the keratan epitope, which therefore represent a distinct subpopulation. The extractabilities of molecules detected by their GAG and keratan sulphate epitope contents are, however, similar in control (about 10\%) and IL- $1 \alpha$ (about $10 \%-15 \%$ ) treated joints (compare fig $4 \mathrm{~A}$ and fig $5 \mathrm{~B}$ ). As described below, extractability of molecules bearing the 846 epitope was not increased in joints treated with IL- $1 \alpha$.

Regression analysis between proteoglycan content and extractability showed that there were inverse relations between total content and extractability within a joint which suggest that increased extractability may reflect increased degradation and resultant loss. Thus in control joints both keratan epitope $(r=-0.57$, $\mathrm{p}<0.05)$ and GAG $(r=-0.68, \mathrm{p}<0.05)$ showed significant inverse relations in the dose response study. For joints injected with IL-1 $\alpha$, extractability of GAG (but not the keratan sulphate epitope) was also inversely related to total content $(\mathrm{p}<0.05, \mathrm{p}=-0.57)$.

AGGRECAN CONTENTS OF SYNOVIAL FLUIDS By 24 hours contents of GAG and keratan sulphate were significantly increased at 10 and $50 \mathrm{ng}$ IL-1 $\alpha$ (fig 5). Whereas this represented about a twofold increase in GAG at $10 \mathrm{ng}$ and a fourfold increase at $50 \mathrm{ng}$ (fig 5D) much greater increases in immunoreactive keratan sulphate were noted, being about 10 -fold at both $10 \mathrm{ng}$ and $50 \mathrm{ng}$ (fig $5 \mathrm{C}$ ).

CHANGES AFTER INJECTION OF $10 \mathrm{ng}$ IL-1 $\alpha$ : TIBIAL MEDIAL CARTILAGE Aggrecan content and extractabilities

Analyses of the time dependent changes in the tibial medial compartment disclosed that total GAG content was decreased at 24 hours, after injection of $10 \mathrm{ng}$ IL-1 $\alpha$, but this decrease was not significant in this experiment until 96 hours (fig 6A). The increased extractability found previously at 24 hours was again noted in this separate study. It was first seen at six hours, and persisted at 96 hours (fig 6B). This correlated inversely with the total content of GAG in control joints $(r=-0.70, \mathrm{p}<0.05)$. Moreover, increased GAG extractability in IL-1 $\alpha$ injected joints was inversely correlated with extractability in contralateral joints $(r=-0.62$, $\mathrm{p}<0 \cdot 05)$. The total keratan sulphate epitope content was not significantly reduced at any time (fig 7A) even at $50 \mathrm{ng} \mathrm{IL-1} \alpha /$ joint (fig $5 \mathrm{~A}$ ). Changes in extractability of immunoreactive keratan sulphate were transient, in that an increase was only noted at 24 hours. Analyses were also made of the 846 epitope located on 

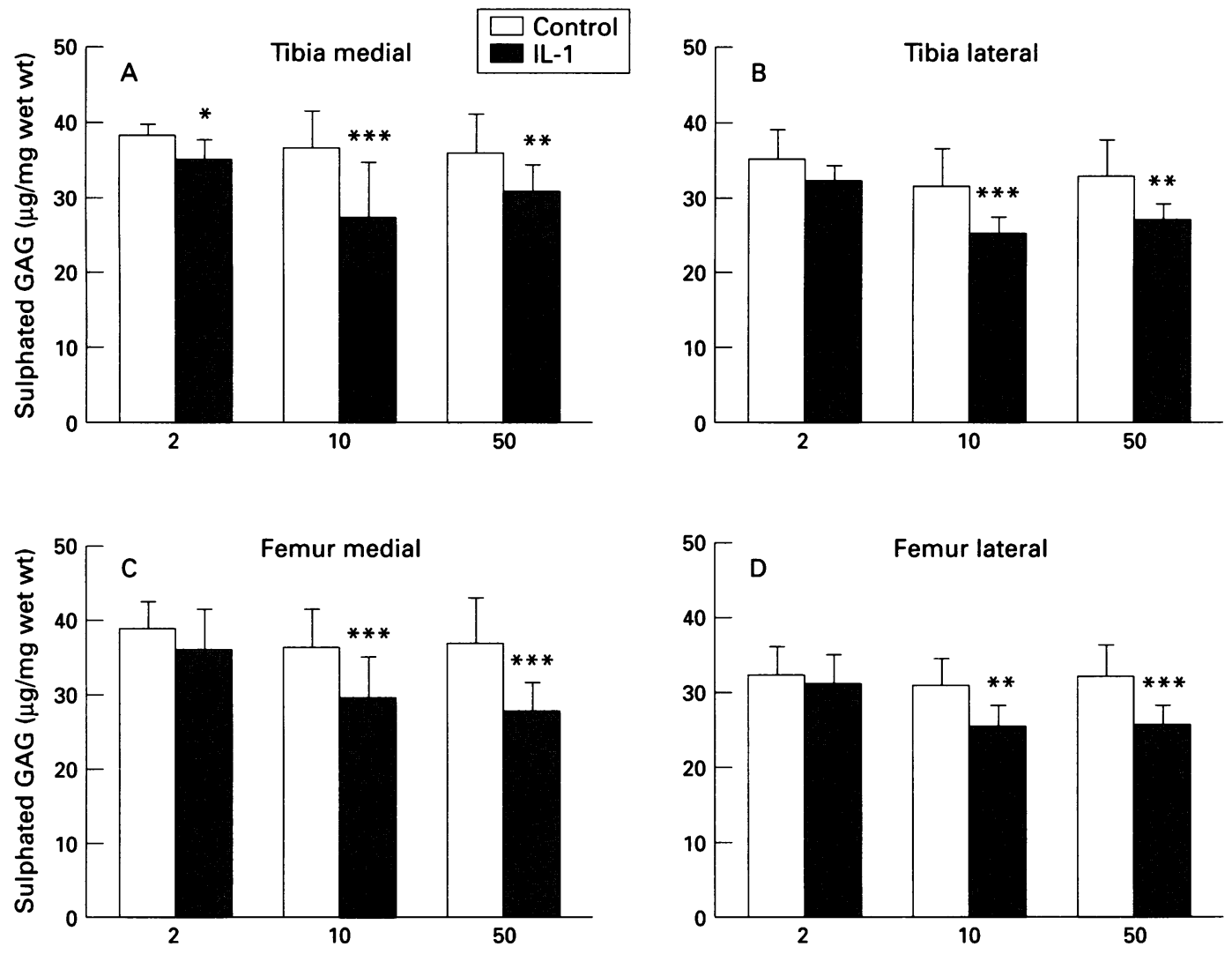

IL-1 (ng/joint)

IL-1 (ng/joint)

Figure 3 Total sulphated GAGs ( $m g$ GAG/mg wet weight cartilage (mean (SD)) in articular cartilage from (A) medial tibia, (B) lateral tibia, (C) medial femur, and (D) lateral femur of the knee joint 24 hours after an intra-articular injection of $B S A$ (control) or 2,10 , and 50 ng. IL-1 with BSA $(n=6)$. ${ }^{*} p<0.01 ; * \star *_{p}<0.001$.

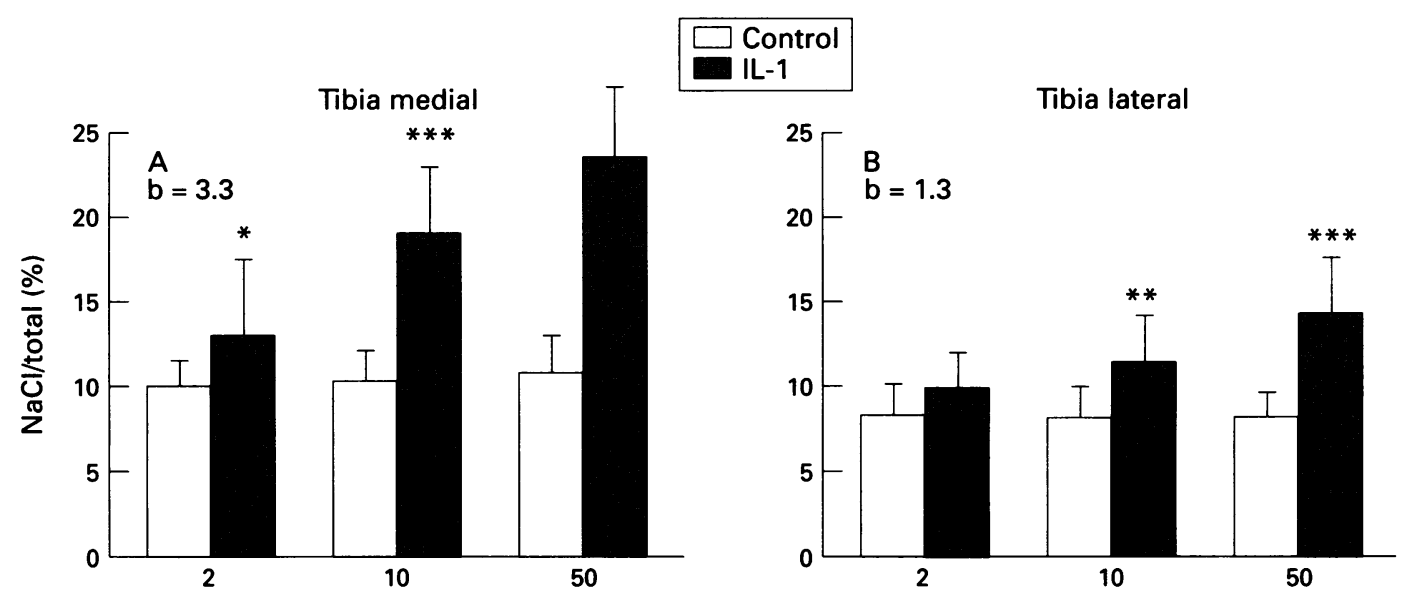

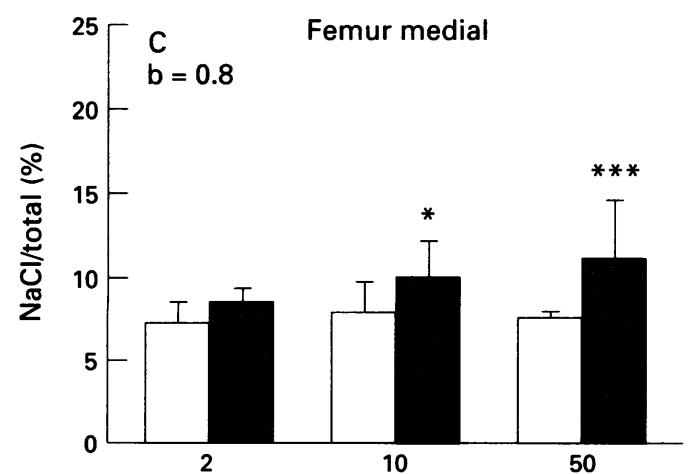

IL-1 (ng/joint)

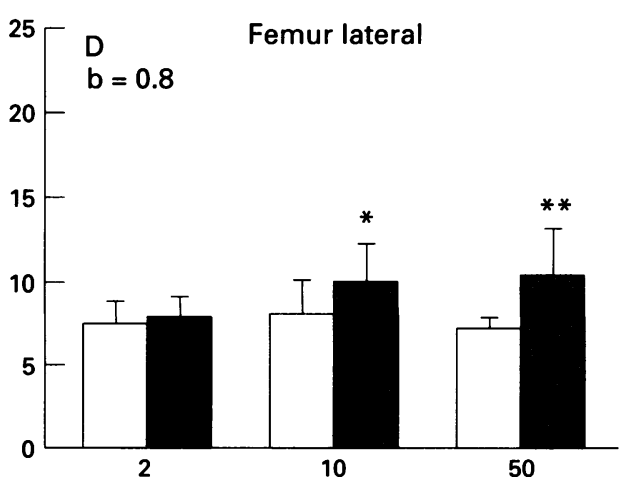

IL-1 (ng/joint)

Figure 4 Extractability of $\mathrm{GAG}$ (in $\mathrm{NaCl}$ extract as \% of total) (mean (SD)) in articular cartilage from (A) medial tibia, (B) lateral tibia, (C) medial femur, and (D) lateral femur of the knee joint 24 hours after an intra-articular injection of $B S A$ (control) or 2,10 , and $50 \mathrm{ng} I L-1$ with $B S A(n=6), b=$ slope of loglinear dose-response relation, $y=a+b x$, where $x=\ln ($ dose $)$. ${ }^{\star} p<0.05 ;{ }^{\star *} p<0.01 ;{ }^{\star *} p<0.001$. 
Total keratan sulphate in cartilage Tibia medial
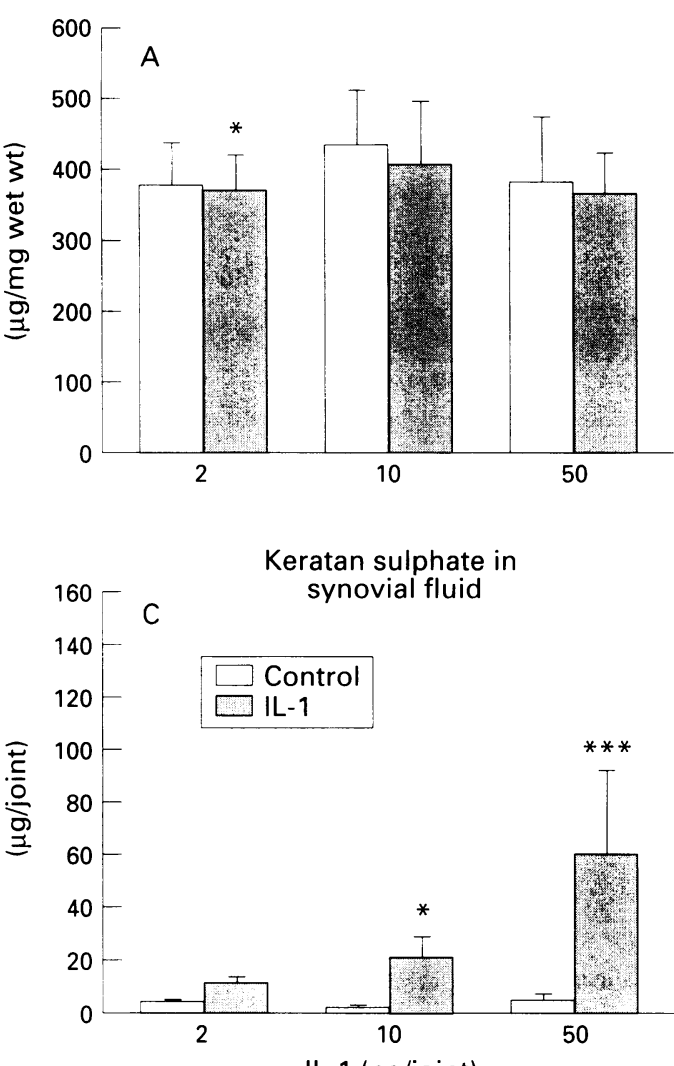

Cartilage keratan sulphate extractability Tibia medial
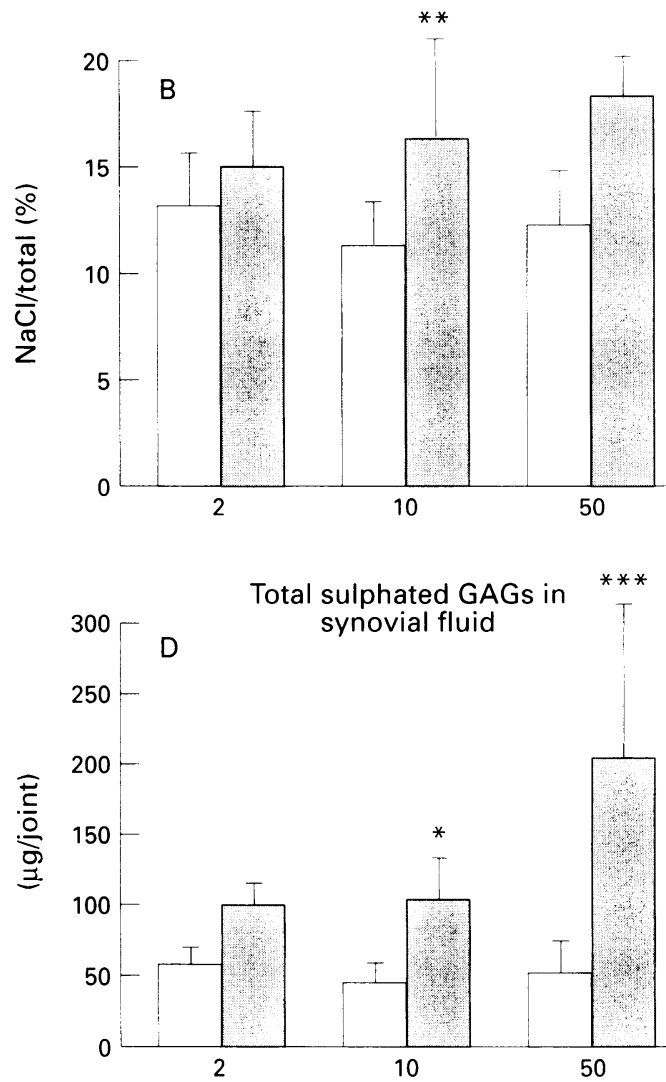

IL-1 (ng/joint)

Figure 5 (A) Total keratan sulphate in medial tibial cartilage, (B) keratan sulphate epitope extractability in medial tibia (NaCl extract as \% of total), (C) total keratan sulphate, and (D) total GAG in synovial lavage fluid of the knee joint at 24 hours after intra-articular injection of $B S A$ (control) or 2,10 , and $50 \mathrm{ng} I L-1$ with BSA $(n=6)($ mean $(S D))$. ${ }^{\star} p<0.05 ;{ }^{\star *} p<0.01 ;{ }^{\star \star *} p<0.001$.

the chondroitin sulphate chains of aggrecan. This exhibited no significant change in content at any time although the total content in the non-IL- $1 \alpha$ injected joint was reduced (but not significantly) at 24 and 96 hours compared with that at six hours (fig $8 \mathrm{~A}$ ). This correlated directly with content in the contralateral joint $(r=0.73, \mathrm{p}<0 \cdot 01)$.

Extractability of molecules bearing the 846 epitope was unchanged with time in joints injected with IL-1 $\alpha$ (fig $8 \mathrm{~B})$. As this was in the range $20 \%-30 \%$ in control joints compared with about $10 \%$ for GAG and immunoreactive karatan sulphate, the molecules bearing this 846 epitope would seem to be less tightly bound. Moreover, in control joints, extractability of 846 epitope was inversely correlated with total content of this epitope $(r=-0.58, \mathrm{p}<0.05)$. The table summarises these changes.

\section{Aggrecan content of synovial fluids}

GAG (fig 6C) and keratan sulphate epitope (fig 7C) were similarly increased (up to fourfold) at six hours and 24 hours. This increase had disappeared by 96 hours. Epitope 846 was also maximal and similarly increased at six and 24 hours (fig 8C) but not at 96 hours. But whereas GAG content was directly correlated between IL- $1 \alpha$ and contralateral joints $(r=0 \cdot 60$, $\mathrm{p}<-0.05)$ an inverse correlation was noted in immunoreactive keratan sulphate $(r=-0 \cdot 85$, $\mathrm{p}<0.01)$. The table summarises these changes.

\section{Discussion}

It has previously been established that intraarticular injection of IL-1 produces an acute infiltration of polymorphonuclear leucocytes into the injected joint, which is accompanied by a loss of proteoglycan from articular cartilage into synovial fluid. ${ }^{13-16}$ This transient increase in cell number was dose dependent and rapid, being more pronounced at six hours and 24 hours and being absent at 96 hours. In the present study we sought to examine the proteoglycan changes in more detail, particularly in view of the recent identification of distinct subpopulations of the proteoglycan aggrecan $^{6}$ which may behave differently. These studies produced the same general findings although there was variation between experiments in terms of the timing of events and quantitative differences, presumably due to individual differences in the response of rabbits. Considerable individual differences between rabbits were also found for the 846 epitope, compared with GAG and the keratan sulphate epitope. Despite these variations, the present experiments have provided some important new insights into aggrecan metabolism in inflamed joints that is altered by injection of IL- $1 \alpha$.

Studies of animal, ${ }^{7}$ and particularly human, cartilages $^{689}$ have drawn attention to the presence of distinct aggrecan subpopulations. The largest molecules are chondroitin sulphate rich and contain the epitope 846. Larger 

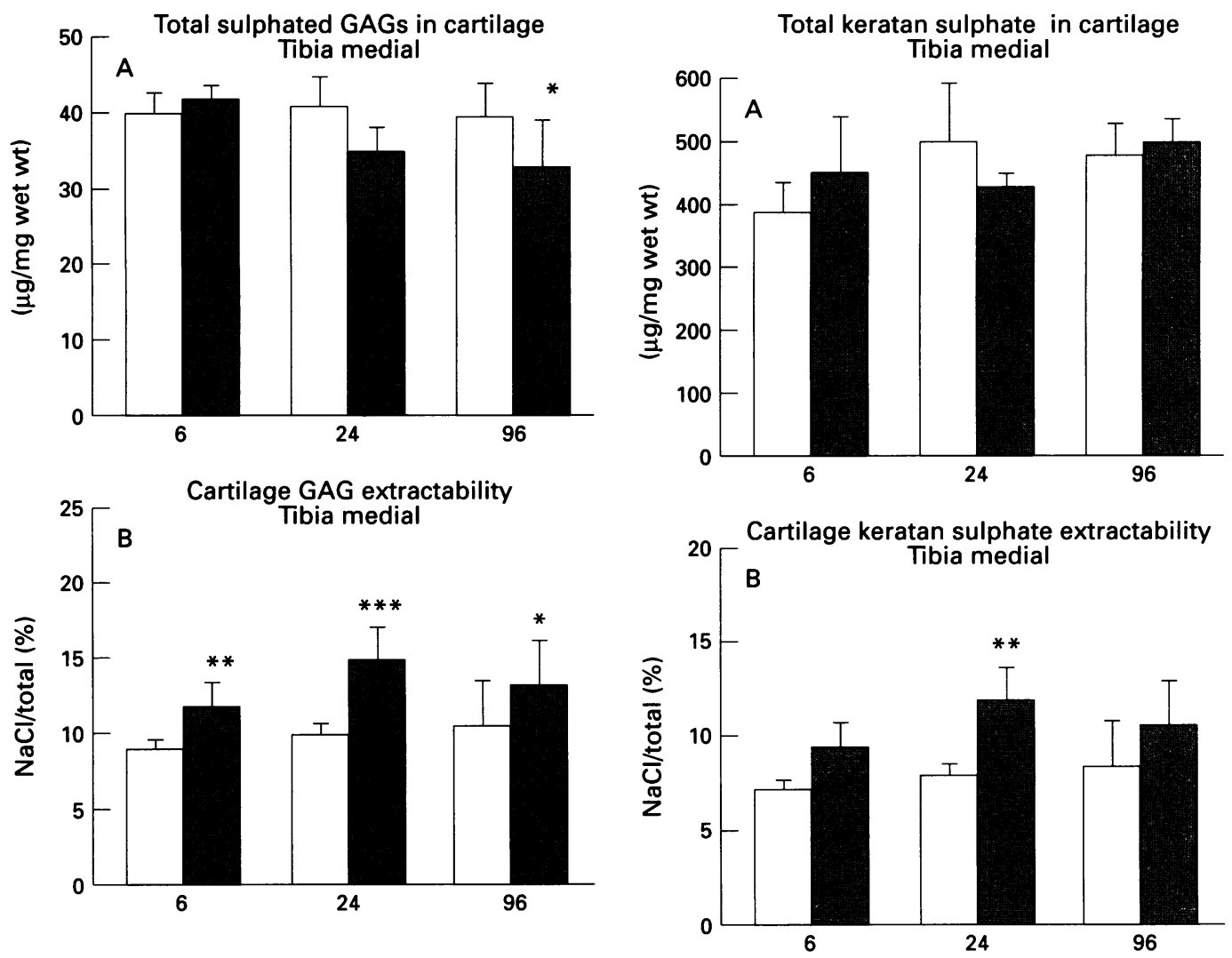

Total sulphated GAGs in synovial fluid

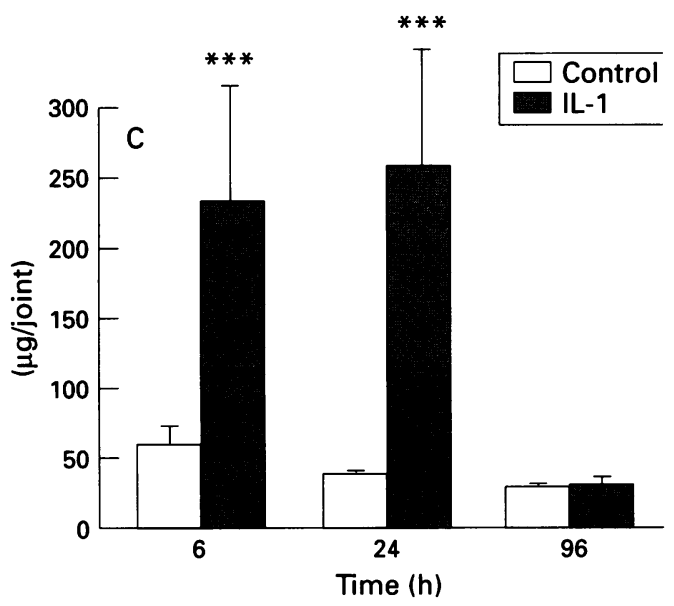

Figure 6 (A) Total sulphated GAGs in medial tibial cartilage, (B) GAG extractability in medial tibial ( $\mathrm{NaCl}$ extract as \% of total) and (C) total sulphated GAGs in synovial lavage fluid of the knee at 6,24 , and 96 hours after an intra-articular injection of $B S A$ (control) or $10 \mathrm{ng}$ IL-1 with BSA $(n=4)$ (mean (SD)). ${ }_{*}<<0.05$; ${ }^{*} p<0.01 ; * * x<0.001$.

molecules bearing this epitope were also found in articular cartilages from these rabbits. But as the immunoassay of these epitopes necessitated using different fetal human and rabbit costal cartilage aggrecan standards (because of their epitope contents, which were either high in keratan sulphate and deficient in 846 epitope or vice versa), and not rabbit articular aggrecan, it was not possible to draw any conclusions concerning the absolute concentrations of rabbit articular aggrecan represented by these different analyses. The analyses were, however, useful in identifying complex and variable changes involving aggrecan molecules not previously identified.

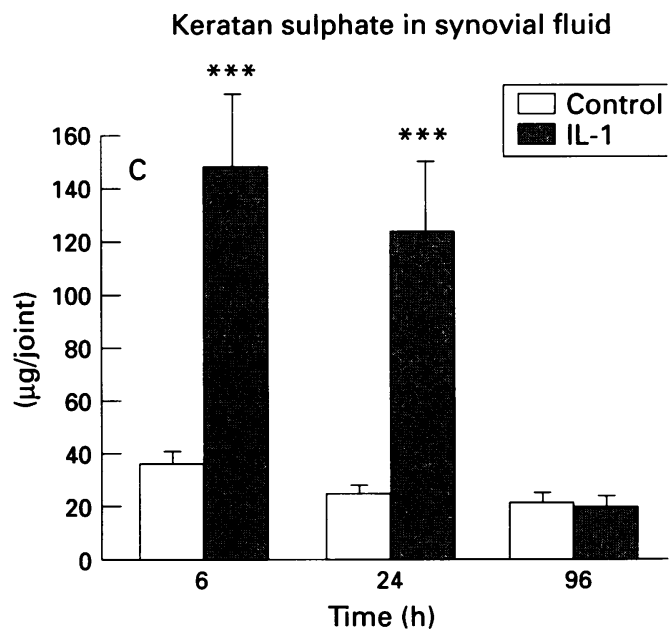

Figure 7 (A) Total keratan epitope in medial tibial cartilage, (B) keratan sulphate epitope extractability in medial tibial cartilage ( $\mathrm{NaCl}$ extract as \% of total), and (C) total keratan sulphate epitope in synovial lavage fluid of the knee joint at 6, 24, and 96 hours after an intraarticular injection of BSA (control) or $10 \mathrm{ng} I \mathrm{~L}-1$ with $B S A(n=4)($ mean $(S D)) . \star_{p}<0.01 ; * \star * p<0.001$.

Further recent in vitro analyses ( $\mathrm{H}$ Jugessur and A R Poole, unpublished data), indicate that aggrecan molecules bearing the 846 epitope represent recently synthesised molecules. Using Spearman rank analyses, we have noted a very significant correlation $(r=0.812$, $\mathrm{p}=0.0004, \mathrm{n}=20$ ) between 846 epitope content in bovine fetal/articular cartilage and aggrecan synthesis measured by ${ }^{35} \mathrm{~S}$-sulphate incorporation into chondroitin sulphate aggrecan. Whereas in osteoarthritic cartilages this 846 epitope is increased in cartilage ${ }^{6}$ when aggrecan synthesis increases, ${ }^{22}{ }^{23}$ no such increase in the epitope was found in the short time period studied after a single injection of 

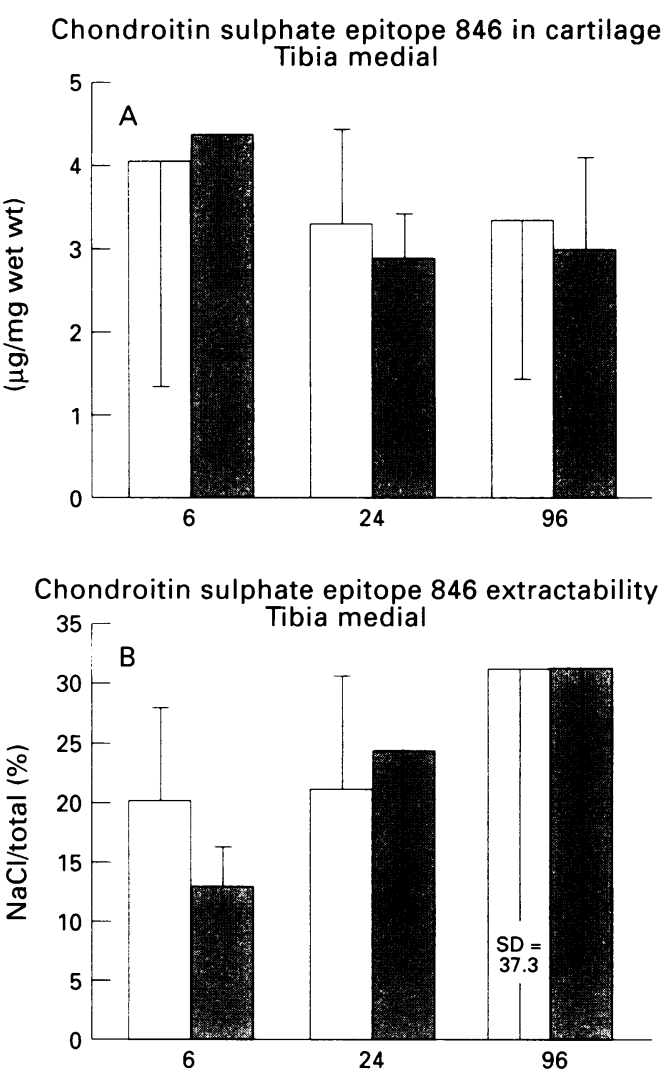

Chondroitin sulphate epitope 846 in synovial fluid

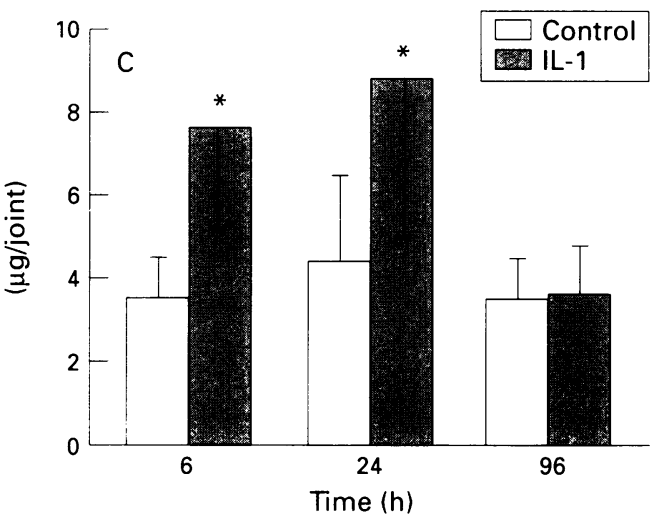

Figure 8 (A) Chondroitin sulphate epitope 846 in medial tibial cartilage (B) 846 exctracability in tibial medial cartilage ( $\mathrm{NaCl}$ extract as \% of total), and (C) total 846 in synovial lavage fluid of the knee joint at 6, 24, and 96 hours after an intra-articular injection of $B S A$ (control) or $10 \mathrm{ng} \mathrm{IL-1}$ with BSA $(n=4)$ (mean (SD)). ${ }^{\star} p<0.05$.

IL-1 $\alpha$. In fact both joints showed evidence of a decrease in this epitope. Although this reduction was not significant within experimental or control knees, it did show significant direct correlations between knees. The lack of increase in this epitope may be because IL-1 can inhibit proteoglycan synthesis both in vitro $^{2425}$ and in vivo). ${ }^{15-17}$ The increases of the 846 epitope as well as the keratan sulphate epitope in synovial fluid suggest that molecules already present in articular cartilage bearing these epitopes are cleaved and released from cartilage in the presence of IL-1 without any detectable change in their content in cartilage.

The mean total cartilage content of these joints is of the order of $90 \mathrm{mg}$ wet weight (C Lundberg et al, unpublished data). Thus if we assume that aggrecan contents are similar in different sites, we can calculate from the data in fig 6 that, in a control joint at six hours the SF content of GAG represents only about $1.6 \%$ of the total cartilage plus fluid content of GAG. After IL- $1 \alpha$ injection, this rises at six and 24 hours to about $7 \cdot 2 \%$ of the total cartilage plus fluid content. Thus these synovial changes involve a limited contribution from the cartilage which is not initially detectable by a reduction in the tissue content, although the increase in synovial fluid is accompanied by an increase in extractability. Moreover, in view of the lack of specificity of GAG for cartilage, some of it may be derived from the synovium. Figure 7 shows that the keratan sulphate content in synovial fluid in normal joints represents even less: it is about $0.11 \%$ of the total cartilage plus fluid content at six hours rising only to $0.32 \%$ of the content at 24 hours after IL- $1 \alpha$ injection. For the 846 epitope (fig 8), this rises in synovial fluid from $1.0 \%$ of the content in the control joint at six hours to $3 \cdot 3 \%$ of the total joint content at 24 hours after IL-1 $\alpha$ injection. Therefore, expressed in this manner, the total content of keratan sulphate and 846 epitopes in synovial fluid represents a relatively small proportion of the total cartilage content of these epitopes in normal and IL- $1 \alpha$ injected joints. This helps to explain the lack of detectable change in cartilage content for these epitopes. Increases in cartilage extractability, are, however, detectable and clearly relate to the increased release into synovial fluid, pointing to the value of such measurements and indicating that the increased extractability reflects increased release of these molecules.

The heterogeneity of changes involving aggrecan molecules shown by these studies points to the fact that different molecules and fragments thereof are undergoing changes induced by $\mathrm{IL}-1 \alpha$. Whereas immunoreactive keratan sulphate molecules may characterise those more typical of the partly degraded "resident" population present throughout the extracellular matrix, those molecules bearing the 846 epitope, which are normally more extractable (mean $20 \%$ of total), may, therefore, be less integrated into the matrix, compared with those molecules bearing the keratan sulphate epitope and those detected by GAG analysis (mean about $10 \%$ of total). Compared with the 846 epitope, the greater release of the keratan sulphate epitope after IL- $1 \alpha$ injection may be explained by keratan sulphate being primarily present on resident molecules. These could be degraded. By contrast the synthesis of molecules bearing the 846 epitopes may be inhibited by IL-1 $\alpha$. This may explain the lack of loss of these molecules. Analyses of GAG molecules clearly showed that IL-1 $\alpha$ could induce a net loss of GAG rich proteoglycan from articular cartilage after injection of $10 \mathrm{ng} \mathrm{IL}-1 \alpha$, which was found at 24 hours or 96 hours depending on the experiment. This was also accompanied or preceded by an increase in GAG extractability. By contrast, a less pronounced increase and more transient increase in extractability was seen for molecules bearing the keratan sulphate 
epitope with no changes for the normally more extractable 846 bearing molecules. The second may also relate to inhibition of synthesis of 846 bearing molecules.

Such increases in extractability have been noted in arthritic cartilages in early experimental osteoarthritis ${ }^{26} 27$ and in human disease $^{28}$ and are thought to reflect collagen damage. In the present studies the inverse relation of total aggrecan content (measured as GAG or keratan sulphate epitope) to extractability seen in both control and experimental joints also points to the relation of extractability with cartilage damage and loss of aggrecan.

The study also serves to highlight the regional differences in the responses of cartilage to $\mathrm{IL}-1 \alpha$ injection after 24 hours. Only the medial tibial compartment exhibited a significant reduction and increased extractability in total GAG content after injection of $2 \mathrm{ng} \mathrm{IL-1} \alpha$. Moreover, extractability at $10 \mathrm{ng} \mathrm{IL-1 \alpha /joint} \mathrm{was} \mathrm{greater} \mathrm{in}$ this compartment. For this reason, the remaining studies concentrated on analyses of this cartilage and $10 \mathrm{ng} /$ joint of $\mathrm{IL}-1 \alpha$. It is well recognised that in early osteoarthritis degenerative changes are usually more pronounced in this tibial compartment, both in experimental and natural disease in animals ${ }^{26} 2729$ and in humans. ${ }^{31}$ The present findings also point to a greater sensitivity of this medial cartilage to IL-1 $\alpha$. Others have also noted that chondrocytes in the medial tibial plateau of mice are more susceptible to II-1 induced suppression of proteoglycan synthesis. ${ }^{30}$ From these results, this sensitivity would seem to extend to degradation. The reasons for the more rapid development of degenerative changes in tibial medial articular cartilage merit further careful study.

In recent years there has been much interest in measurement of proteoglycan fragments and GAG in synovial fluids as they may reflect alterations of cartilage metabolism within the joint. $^{18193233}$ The present study of changes in both the joint cartilage and synovial fluid of the same joint with time after injection of $10 \mathrm{ng}$ IL-1 $\alpha$ offers some new insights into these relations which have not previously been available from human studies. The increases in GAG, keratan sulphate epitope, and 846 epitope in synovial fluid after injection of $10 \mathrm{ng}$ IL- $1 \alpha$ were all transient, occurring at six hours and 24 hours and, returning to normal at 96 hours. Thus they coincided with the increase in leucocyte content, which was not seen at 96 hours. Clearly, as discussed above, unlike the GAG changes, the increased release of cartilage proteoglycan bearing keratan sulphate and 846 epitopes into synovial fluid occurred in the absence of changes in the contents of these epitopes in articular cartilage, and only a transient increase in extractability of the keratan sulphate epitope at 24 hours. Thus the changes in synovial fluid sensitively reflect subtle changes in cartilage metabolism.

In conclusion, this study provides new insight into the heterogeneity of changes involving the proteoglycan aggrecan in articular cartilages after exposure to a proinflammatory cytokine. It shows that changes in cartilage and joint fluids are not always related, that synovial fluid measurements of defined aggrecan epitopes alone can provide new sensitive insights into changes in aggrecan metabolism, and reflect the response of articular cartilage to damage by proinflammatory molecules such as IL-1, which may not otherwise be apparent if cartilage alone is analysed. The findings lend support to the use of analyses of synovial fluid in studies of joint diseases, which have become common practice in recent studies of patients with rheumatoid arthritis and osteoarthritis. ${ }^{19}$ 32-36

We are grateful to Dr Margareta Holgersson, Pharmacia Therapeutics, for the statistical evaluations. The work was funded by Pharmacia, Shriners of North America (ARP), and the Medical Research Council of Canada (ARP).

1 Poole A R. Cartilage in health and disease. In: McCarty D J, Koopman $W$ J, in Arthritis and allied conditions. A textbook of rheumatology. 12th ed. Philadelphia: Lea and textbook of rheumatology.
Febiger, 1993; 279-333.

2 Heinegård $D$, Oldberg $\AA$. Structure and biology of cartilage and bone matrix non-collagenous macromolecules. FASEB F 1989; 3: 2042-51.

3 Doege K J, Sasaki M, Kimura T, Yamada Y. Complete coding sequence and deduced primary structure of the human cartilage large aggregating proteoglycan aggrecan. Human-specific repeats and additional alternatively spliced forms. F Biol Chem 1991; 266: 894-902.

4 Doege K, Sasaki M, Horigan E, Hassell J R, Yamada Y. Complete primary structure of the rat cartilage proteoglycan core protein deduced from cDNA clones. $f$ proteoglycan core protein deduced

5 Glant T T, Mikecz K, Roughley P J, Buzas E, Poole A R. Age-related changes in protein-related epitopes of human articular-cartilage proteoglycans. Biochem $\mathcal{f}$ 1986; 236: 71-5.

6 Rizkalla G, Reiner A, Bogoch E, Poole A R. Studies of the articular cartilage proteoglycan aggrecan in health and osteoarthritis. Evidence for molecular heterogeneity and extensive molecular changes in disease. $\mathcal{F}$ Clin Invest 1992; 90: 2268-77.

7 Franzen A, Inerot S, Bejderuf S O, Heinegård D. Variations in the composition of bovine hip articular cartilage with distance from the articular surface. Biochem $f$ 1981; 195: 535-43.

8 Bayliss $M$ T, Ali S Y. Age-related changes in the composition and structure of human articular cartilage proteoglycans. Biochem $\mathcal{\Im} 1978 ; 176: 683-93$.

9 Webber C, Glant T T, Roughley P J, Poole A R. The identification and characterization of two populations of aggregating proteoglycans of high buoyant density isolated from post-natal human articular cartilages of different ages. Biochem $\mathcal{f}$ 1987; 248: 735-40.

10 Sandy J D, Neame P J, Boynton R E, Flannery C R. Catabolism of aggrecan in cartilage explants. Identification of a major cleavage site within the interglobular domain. F Biol Chem 1991; 266: 8683-5.

11 Loulakis P, Shrikharde A, Davis G, Maniglia C A. $\mathrm{N}$-terminal sequence of proteoglycan fragments isolated cultures. Putative site(s) of enzymic cleavage. Biochem $\mathfrak{f}$ cultures. Putative sice

12 Sandy J D, Flannery C R, Neame P J, Lohmander L S. The structure of aggrecan-fragments in human synovial fluid. Structure of aggrecan-fragments in human synovial fluid. Evidence for the involvement in osteoarthritis of a novel-
proteinase which cleaves the Glu 373-Ala 374 bond of the interglobular domain. $\mathcal{f}$ Clin Invest 1992; 89: 1512-6.

13 Pettipher E R, Higgs G A, Henderson B. Interleukin 1 induces leukocyte infiltration and cartilage proteoglycan degradation in synovial joint. Proc Natl Acad Sci USA 1986; 83: 8749-53

14 Pettipher E R, Henderson B, Hardingham T, Ratcliffe A. Cartilage proteoglycan depletion in acute and chronic antigen-induced arthritis. Arthritis Rheum 1989; 32: 601-7.

15 van de Loo A A J, van den Berg W B. Effects of murine recombinant interleukin-1 on synovial joints in mice: recombinant interleukin-1 on synovial joints in mice: measurement of patellar cartilage metabolism and

16 Chandrasekhar S, Harvey A K, Hrubey P S. Intra-articular administration of interleukin-1 causes prolonged suppression of cartilage proteoglycan synthesis. Matrix 1992; 11: 1-10.

17 van de Loo F A J, Arntz O J, Otterness I G, van den Berg W. Protection against cartilage proteoglycan synthesis inhibition by anti-interleukin-1 antibodies in experimental arthritis. $\mathcal{F}$ Rheumatol 1992; 19: 348-56.

8 Witter J, Roughley P J, Webber C, Roberts N, Keystone E, Poole A R. The immunological detection and characterization of cartilage proteoglycan degradation products in synovial fluids of patients with arthritis. Arthritis Rheum 1987; 30: 519-26.

19 Poole A R, Ionescu M, Swan A, Dieppe P. Changes in cartilage metabolism in arthritis are reflected by altered glycan aggrecan: implications for pathogenesis. f Clin Invest 1994; 94: 25-33. 
20 Farndale R W, Buttle D J, Barrett A J. Improved quantitation and discrimination of sulphated glycosaminoglycans by use of dimethylmethylene blue. Biochim Biophys Acta 1986; 883: 173-7.

21 Poole A R, Reiner A, Roughley P J, Champion B. Rabbit antibodies to degraded and intact glycosaminoglycans which are naturally occurring and present in arthritic which are naturally occurring and prese
rabbits. $f$ Biol Chem 1985; 260: 6020-5.

22 Mankin H J, Lipiello L. Biochemical and metabolic abnormalities in articular cartilage from osteoarthritic human hips. F Bone foint Surg Am 1970; 52: 424-34

23 Thompson R, Oegema T. Metabolic activity of articular cartilage in osteoarthritis. $\mathcal{F}$ Bone foint Surg Am 1979; 61: 407-16.

24 Tyler J A. Articular cartilage cultured with catabolin (pig interleukin 1) synthesizes a decreased number of proteoglycan molecules. Biochem $\mathcal{f}$ 1985; 227: 869-78.

25 Arner E C, Pratta M A. Independent effects of interleukin-1 on proteoglycan breakdown, proteoglycan synthesis and prostaglandin $\mathrm{E}_{2}$ release from cartilage in organ culture. prostaglandin $\mathrm{E}_{2}$ release from car

26 McDevitt C, Gilbertson E, Muir H. An experimental model of osteoarthritis: early morphological and biochemical changes. F Bone foint Surg 1977; 59-B: 24-35.

27 McDevitt C, Muir H. Biochemical changes in the cartilage of the knee in experimental and natural osteoarthrosis in the dog. F Bone foint Surg 1976; 58-B: 94-101.

28 Brandt K D. Enhanced extractability of articular cartilage proteoglycans in osteoarthritis. Biochem $f$ 1974; 143:
29 Schünke $M$, Tillmann B, Brück $M$, Müller-Ruchholtz W. Morphologic characteristics of developing osteoarthritis lesions in the knee cartila
Rheum 1988; 31: 898-905.

30 van Beuningen $\mathrm{H}$ M, Arntz O J, van den Berg W B. In vivo effects of interleukin-1 on articular cartilage. Prolongation of proteoglycan metabolic disturbances in old mice. Arthritis Rheum 1991; 34: 606-15.

31 Dean D D, Martel-Pelletier J, Pelletier J-P, Howell D S, Woessner J F. Evidence for metalloproteinase-inhibito mbalance in human osteoarthritic cartilage. 7 Clin Invest 1989; 84: 678-85.

32 Ratcliffe A, Doherty M, Maini R N, Hardingham T E. Increased concentrations of proteoglycan components in the synovial fluids of patients with acute but not chronic joint disease. Ann Rheum Dis 1988; 47: 826-32.

33 Saxne T, Heinegård D. Synovial fluid analysis of two groups of proteoglycan epitopes distinguishes early and later of proteoglycan epitopes distinguishes early and
cartilage lesions. Arthritis Rheum 1992; 35: 385-90.

34 Mansson B, Carey D, Alini M, et al. Cartilage and bone metabolism in rheumatoid arthritis. Differences between rapid and slow progression of disease identified by serum markers of cartilage metabolism. F Clin Invest 1995; 95 1071-7.

35 Poole A R. Immunochemical markers of joint inflammation, skeletal damage and repair: where are we now? Ann Rheum Dis 1994; 53: 3-5.

36 Poole A R, Dieppe P D. Biological markers in rheumatoid arthritis. Semin Arthritis Rheum 1994; 23 (suppl 2): $17-31$. 